\title{
Exact Calculation of Ellipse Perimeter by Analytical Method
}

\author{
Abolfazl Soltani * \\ Department of Physics, University of Birjand, Birjand, Iran
}

Email: soltani.a.physics@gmail.com

\section{Abstract}

As you know, we do not have any exact equation for calculating the perimeter of an ellipse. In this article, we obtain this equation analytically.

Keywords: Ellipse perimeter, Ellipse, Area of ellipsoid

\section{Introduction}

Consider the following equation:

$$
r=\frac{e d}{1-e \operatorname{Cos} \theta}
$$

This equation is the equation of a conic section in the polar coordinate system, which is obtained using the directrix-focus property of conic sections based on Figure 1 [1],[2].

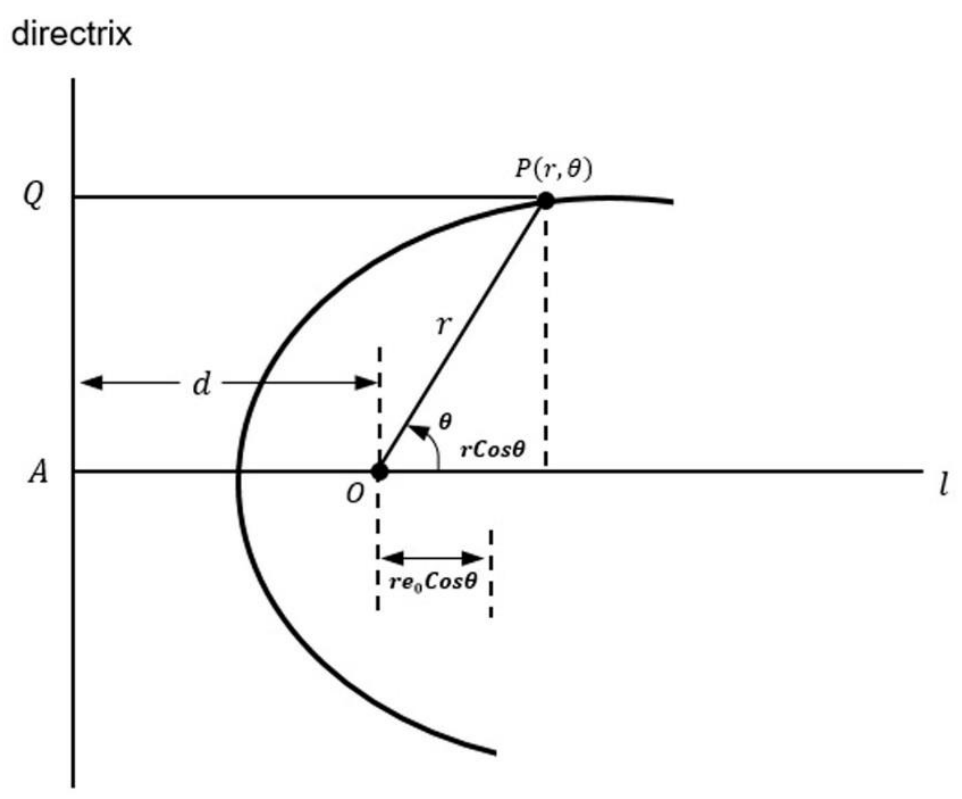

Fig. 1. This figure shows an ellipse as a conic section. Based on the directrix-focus property, we have: $|Q P| /|O P|=e$. In an ellipse because of $0<e<1$ we have: $r_{0} \operatorname{Cos} \theta<r \operatorname{Cos} \theta$, which you can observe it in the figure.

In equation $1, d$ is the distance between a conic section directrix line and the focal point close to it [1],[2]. For an ellipse with Semi major axis $a=a_{0}$ and $e=e_{0}$ we have: $d=a_{0} / e_{0}-e_{0} a_{0}$ [1]. substituting $d$, equation 1 for this ellipse is as follows [1]: 


$$
r=\frac{a_{0}\left(1-e_{0}^{2}\right)}{1-e_{0} \operatorname{Cos} \theta}
$$

Now we want to obtain the ellipse perimeter using equation 2. Using the formula

$$
L=\int_{\alpha}^{\beta} \sqrt{r^{2}+\left(\frac{d r}{d \theta}\right)^{2}} d \theta
$$

Which is the equation of calculation of the length of a polar curve [1],[3] and according to equation 2, we have:

$$
L_{\text {Ellipse }}=a_{0}\left(1-e_{0}^{2}\right) \int_{0}^{2 \pi} \sqrt{\frac{\left(1-e_{0} \operatorname{Cos} \theta\right)^{2}+e_{0}^{2} \operatorname{Sin}^{2} \theta}{\left(1-e_{0} \operatorname{Cos} \theta\right)^{4}}} d \theta
$$

As you can see, the above integral is clearly difficult to solve (probably the above integral is a kind of elliptic integral). So we need to find the perimeter of the ellipse in another way. First we write equation 2 as follows:

$$
r-r e_{0} \operatorname{Cos} \theta=a_{0}\left(1-e_{0}^{2}\right)
$$

If we consider the left side of equation 5 equal to $\eta$, then we have

$$
\eta=a_{0}\left(1-e_{0}^{2}\right)
$$

It is clear, in the above equation, $\eta$ is a function of $\theta: \eta=\eta(\theta)$ like $r=r(\theta)$. Inasmuch as $r^{2}\left(\operatorname{Sin}^{2} \theta+\operatorname{Cos}^{2} \theta\right)=a^{2}$ or $r=a$ is the equation of a circle, we can conclude that equation 6 is the equation of a circle with radius $a_{0}\left(1-e_{0}{ }^{2}\right)$. This means that equation 2 is both the equation of an ellipse and the equation of a circle. So to calculate the perimeter of an ellipse, we compute the perimeter of its equivalent circle namely equation 6 , instead of calculating integral 4 . Using integral 3 and equation 6 , we have:

$$
L_{\text {Ellipse }}=L_{\text {Equivalent Circle }}=\int_{0}^{2 \pi} \eta d \theta=2 \pi a_{0}\left(1-e_{0}{ }^{2}\right)=2 \pi a_{0}-2 \pi a_{0} e_{0}^{2}
$$

The above equation is our ellipse perimeter equation. If you look at equation 7 , according to figure 2 , you will see that the first sentence of the right side (namely $2 \pi a_{0}$ ), is the perimeter of a circle with radius $a_{0}\left(L_{c_{2}}\right)$. This circle surrounds the ellipse as shown in figure 2 , and its center coincides with the center of the ellipse. Equation 7 shows that the perimeter of the ellipse is less than the perimeter of the circle $C_{2}$, as expected. 


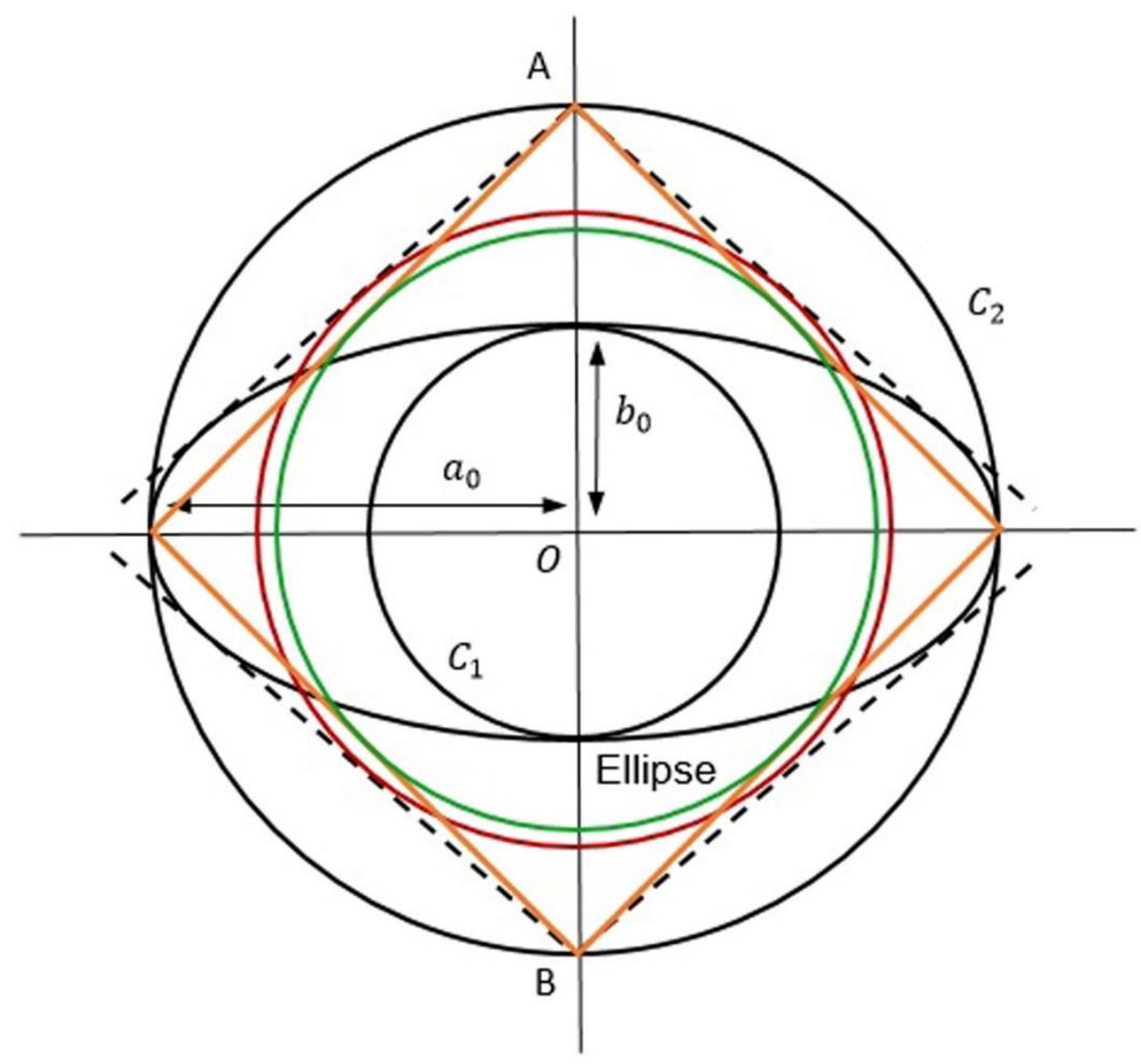

Fig. 2.

We can write equation 7 in another way

$$
b_{0}=a_{0} \sqrt{1-e_{0}^{2}} \Rightarrow L_{\text {Ellipse }}=2 \pi \frac{b_{0}^{2}}{a_{0}}
$$

Where $b_{0}$ is the Semi minor axis of the ellipse.

Where is the location of $C_{\text {equivalent }}$ in Fig. 2? In an ellipse we have: $0<e_{0}<1$. Therefore:

$$
\begin{gathered}
a_{0} \sqrt{1-e_{0}^{2}}<a_{0}\left(1-e_{0}{ }^{2}\right)<a_{0} \\
\stackrel{\times 2 \pi}{\Longrightarrow} 2 \pi a_{0} \sqrt{1-e_{0}^{2}}<2 \pi a_{0}\left(1-e_{0}{ }^{2}\right)<2 \pi a_{0} \Rightarrow L_{c_{1}}<\left(L_{c_{\text {equivalent }}}=L_{\text {Ellipse }}\right)<L_{c_{2}}
\end{gathered}
$$

As shown in Figure 2, four tangent lines to the ellipse can be drawn from points A and B. These four lines are also tangent to the red circle. On the other hand, there is a green circle that is tangent to the orange square faces. As shown in Figure 2, the perimeters of both green and red circles are greater than the perimeter of circle $C_{1}$ and less than the perimeter of circle $C_{2}$. So the inequality 9 is true about them, and therefore, probably, one of the red or green circles is the circle $C_{\text {equivalent }}$. 
Of course, maybe none of them. Many other circles can be drawn with center of $O$ to satisfy inequality 9 . We only guessed here that maybe one of the two green and red circles is our circle $C_{\text {equivalent. }}$

Finally, I need to point out that the area of the circle $C_{\text {equivalent }}$ and its corresponding ellipse are not equal:

$$
A_{E}=\pi a_{0} b_{0}=\pi a_{0}^{2} \sqrt{1-e_{0}^{2}} \quad \text { and } \quad A_{C_{\text {equivalent }}}=\pi \eta^{2}=\pi a_{0}^{2}\left(1-e_{0}^{2}\right)^{2} \quad \Rightarrow A_{C_{\text {equivalent }}} \neq A_{E}
$$

\section{Conclusion}

It seems that after more than 300 years, we have been able to obtain the exact equation of the perimeter of an ellipse. I think using the method of this article can also lead us to the exact equation of the area of an ellipsoid.

\section{References:}

[1]. Silverman, R. Calculus with Analytic Geometry (Prentice-Hall, Inc. New Jersey. 1985), pp. 628-641

[2]. Johnson, R. Calculus with Analytic Geometry (Allyn and Bacon. Inc. Boston, ed. 4), pp. 536-539

[3]. Sherman, S. Calculus with Analytic Geometry (McGraw-Hill, Inc., ed. 3, 1982), pp. 548-554 